\title{
Free sialic acid storage disease
}

INSERM

\section{Source}

INSERM. (1999). Orphanet: an online rare disease and orphan drug data base. Free sialic acid storage disease. ORPHA:834

Free sialic acid storage disease (free SASD), is a group of lysosomal storage diseases characterized by a spectrum of clinical manifestations including neurological and developmental disorders with severity rang ing from the milder phenotype, Salla disease (SD), to the most severe phenotype, infantile free sialic acid storage disease (ISSD). 